\title{
Contrast-Enhanced Time-Resolved MRA for Follow-Up of Intracranial Aneurysms Treated with the Pipeline Embolization Device
}

\author{
S.R. Boddu, F.C. Tong, S. Dehkharghani, J.E. Dion, and A.M. Saindane
}

\begin{abstract}
BACKGROUND AND PURPOSE: Endovascular reconstruction and flow diversion by using the Pipeline Embolization Device is an effective treatment for complex cerebral aneurysms. Accurate noninvasive alternatives to DSA for follow-up after Pipeline Embolization Device treatment are desirable. This study evaluated the accuracy of contrast-enhanced time-resolved MRA for this purpose, hypothesizing that contrast-enhanced time-resolved MRA will be comparable with DSA and superior to 3D-TOF MRA.
\end{abstract}

MATERIALS AND METHODS: During a 24-month period, 37 Pipeline Embolization Device-treated intracranial aneurysms in 26 patients underwent initial follow-up by using 3D-TOF MRA, contrast-enhanced time-resolved MRA, and DSA. MRA was performed on a 1.5T unit by using 3D-TOF and time-resolved imaging of contrast kinetics. All patients underwent DSA a median of 0 days (range, 0-68) after MRA. Studies were evaluated for aneurysm occlusion, quality of visualization of the reconstructed artery, and measurable luminal diameter of the Pipeline Embolization Device, with DSA used as the reference standard.

RESULTS: The sensitivity, specificity, and positive and negative predictive values of contrast-enhanced time-resolved MRA relative to DSA for posttreatment aneurysm occlusion were $96 \%, 85 \%$, 92\%, and $92 \%$. Contrast-enhanced time-resolved MRA demonstrated superior quality of visualization $(P=.0001)$ and a higher measurable luminal diameter $(P=.0001)$ of the reconstructed artery compared with 3D-TOF MRA but no significant difference compared with DSA. Contrast-enhanced time-resolved MRA underestimated the luminal diameter of the reconstructed artery by $0.965 \pm 0.497 \mathrm{~mm}(27 \% \pm 13 \%)$ relative to DSA.

CONCLUSIONS: Contrast-enhanced time-resolved MRA is a reliable noninvasive method for monitoring intracranial aneurysms following flow diversion and vessel reconstruction by using the Pipeline Embolization Device.

ABBREVIATIONS: CE-TR = contrast-enhanced time-resolved; PED = Pipeline Embolization Device; TR-MRA = time-resolved MRA

S urgical clipping or endovascular coil embolization is generally the preferred treatment for intracranial aneurysms. ${ }^{1}$ The Pipeline Embolization Device (PED; Covidien, Irvine, California) is an endovascular device that has redefined the scope of treatment for large, giant, wide-neck, or fusiform aneurysms or aneurysms having failed coil embolization, by reconstructing the parent artery and restoring its natural course. ${ }^{2}$ The PED is designed

Received January 6, 2014; accepted after revision March 31.

From the Departments of Radiology and Imaging Sciences (S.R.B., F.C.T., S.D., J.E.D., A.M.S.) and Neurological Surgery (F.C.T., J.E.D.), Emory University School of Medicine, Atlanta, Georgia.

Paper previously presented as a poster at: Annual Meeting of the American Society of Neuroradiology, May 18-23, 2013; San Diego, California; and as an oral presentation at: 25th International Workshop on Magnetic Resonance Angiography, August 20-23, 2013; New York, New York.

Please address correspondence to Srikanth R. Boddu, MSc, FRCR, Department of Radiology and Imaging Sciences, Division of Neuroradiology, Emory University Hospital, 1364 Clifton Rd NE, BG22, Atlanta, GA, 30322; e-mail: sboddu6@gmail.com http://dx.doi.org/10.3174/ajnr.A4008 for $85 \%$ reduction of blood flow within an aneurysm, which induces thrombosis ${ }^{2}$ while keeping perforators and/or side branch vessels patent. ${ }^{3}$ Results from a multicenter prospective trial for treatment of uncoilable or failed large and giant ICA aneurysms with the PED demonstrated 99\% technical success and 74\% complete occlusion with $6 \%$ major ipsilateral stroke or death. ${ }^{4}$ Flow diversion with the PED has also been reported in the treatment of HIV vasculopathy, with fusiform cerebral aneurysms precluding parent vessel sacrifice or surgical bypass. ${ }^{5}$

Digital subtraction angiography is the reference standard for the evaluation of aneurysms after endovascular treatment due to its unsurpassed spatial resolution; however, DSA is invasive and not without risks of puncture site and neurologic complications. ${ }^{6}$ Posttreatment follow-up of intracranial aneurysms after coil embolization with MRA by using 3D-TOF or contrast-enhanced techniques is a potential noninvasive alternative to DSA for the evaluation of PED-treated aneurysms without the use of ionizing radiation. ${ }^{7}$ Contrast-enhanced time-resolved MRA (CE-TR 
MRA) uses acquisition schemes aimed at accelerated data collection, primarily using parallel imaging algorithms and novel $k$ space trajectories to achieve high temporal resolution for multiphasic MRA examinations. This technique provides consistent, technologist-independent, optimal arterial enhancement for contrast-enhanced MRA and provides information on temporal contrast kinetics. ${ }^{8,9}$ Use of CE-TR MRA has been reported in the evaluation of intracranial lesions such as arteriovenous malformations and dural arteriovenous fistulas and for assessment of stenosis of the extracranial carotid artery, and it seems to be a promising technique for evaluating aneurysms after stent-assisted coil embolization..$^{9-11}$

We hypothesized that TR-CE MRA could provide information comparable with DSA and would be superior to $3 \mathrm{D}-\mathrm{TOF}$ MRA in the evaluation of intracranial aneurysms and the parent artery following flow diversion and parent vessel reconstruction with the PED.

\section{MATERIALS AND METHODS}

\section{Patient and Aneurysm Characteristics}

Approval for this study was obtained from the institutional review board. Since the use of PED at our institution was instituted in April 2011, 27 patients had follow-up evaluation with 3D-TOF MRA, CE-TR MRA, and DSA following PED treatment, allowing direct comparison. One patient with a PED-treated supraclinoid ICA aneurysm was excluded from this study due to extensive susceptibility artifacts on MRA related to a previously clipped contralateral MCA aneurysm, rendering the study nondiagnostic. Of the remaining 26 patients, there were 4 men and 22 women with a median age of 58 years (range, 41-84 years) with 36 aneurysms. The distribution of aneurysms is as follows: cavernous ICA ( $n=$ $10)$, supraclinoid ICA $(n=8)$, ophthalmic artery $(n=7)$, posterior communicating artery $(n=5)$, superior hypophyseal artery $(n=2)$, anterior choroidal artery $(n=2)$, anterior temporal artery $(n=1)$, intracranial vertebral artery $(n=1)$, and carotid terminus $(n=1)$. Eight patients had 2 aneurysms each, and 1 patient had 3 aneurysms. Eighteen patients had a single PED placed, 5 patients had 2 PEDs placed, and 3 patients had 3 PEDs placed. The mean maximal diameter of the aneurysms was $11 \pm$ $7.4 \mathrm{~mm}$ (range, 1.2-28 mm). The median MRA follow-up was 149 days after PED placement (range, 49-184 days). The median time from CE-TR MRA to DSA for follow-up was 0 days (range, 0-68 days) because 19/26 patients had both CE-TR MRA and DSA on the same day.

\section{MRA Technique}

MRA was performed on a 1.5T MR imaging system (Signa HDx; GE Healthcare, Milwaukee, Wisconsin) with an 8-channel head coil. 3D-TOF MRA was first performed by using a gradient-echo sequence (spoiled gradient-recalled-echo) with parallel imaging and scan parameters of TE, $6.9 \mathrm{~ms}$; TR, $38 \mathrm{~ms}$; flip angle, $20^{\circ}$; total acquisition time, 6:09 minutes; number of sections, 110; section thickness, $0.7 \mathrm{~mm}$; FOV, $220 \mathrm{~mm}$; rectangular FOV, 70\%; acquisition matrix, 320; reconstruction matrix, 512; reconstructed voxel size, $0.43 \times 0.43 \times 0.7 \mathrm{~mm}$.

The CE-TR MRA method used in this study is a commercially available sequence (time-resolved imaging of contrast kinetics or
TRICKS; GE Healthcare). Imaging parameters were as follows: TR/TE, 4.5/1.97 ms; flip angle, $35^{\circ}$; FOV, $170 \mathrm{~mm}$; section thickness, $1.2 \mathrm{~mm}$ interpolated to $0.6 \mathrm{~mm}$; reconstructed voxel size, $0.48 \times 0.48 \times 0.7 \mathrm{~mm}$; matrix, $256 \times 160$ zero-filled and interpolated to $512 \times 512$; averages, 0.75 ; bandwidth, $62.5 \mathrm{KHz}$; array spatial sensitivity encoding technique acceleration factor, 2 , yielding 10 or 12 dynamic, temporally discrete postcontrast phases at an approximately 4.0-second temporal update. Time-resolved MRA (TR-MRA) in all patients was performed after the injection of either a low protein-bound gadobenate dimeglumine (MultiHance; Bracco Diagnostics, Princeton, New Jersey), prescribed at $0.1-\mathrm{mmol} / \mathrm{kg}$ and $2-\mathrm{mL} / \mathrm{s}$ injection rates, or the blood pool agent gadofosveset trisodium (Ablavar; Lantheus Medical Imaging, North Billerica, Massachusetts) at $0.03-\mathrm{mmol} / \mathrm{kg}$ and $1.5-\mathrm{mL} / \mathrm{s} \mathrm{injec-}$ tion rates. Bolus infusion in both protocols was followed immediately by $25-$ to $30-\mathrm{mL}$ normal saline flush at $2 \mathrm{~mL} / \mathrm{s}$. Postinjection delay and sequence triggering were prescribed in an automated fashion, without user input. The dynamic data generated by the timeresolved imaging of contrast kinetics sequence are presented in numbered temporal datasets. The scan baseline reflects the simultaneous initiation of the sequence and initiation of contrast injection, both occurring under fully automated parameters prescribed by the scanner/sequence manufacturer without user input. Before contrast/sequence triggering, a noncontrast mask is acquired for subtraction. Background subtraction of the precontrast imaging volume was performed in-line during acquisition, allowing production of both background-subtracted and unsubtracted TR-MRA volumes.

\section{DSA Technique}

All DSA was performed transfemorally with $5 \mathrm{~F}$ catheters by using a DSA unit (Integris Allura; Philips Healthcare, Best, the Netherlands) with an image-intensifier matrix of $1024 \times 1024$ pixels. DSA was performed with bilateral selective internal carotid artery injections and either unilateral or bilateral vertebral artery injections, as necessary. Ten milliliters of nonionic contrast medium (270 mg of iodine per milliliter of iodixanol, Visipaque 270; GE Healthcare) was used for each hand injection. Rotational 3D angiography was performed for additional confirmation of findings. Standard anteroposterior and lateral projections were routinely acquired for carotid and vertebral injections. Additional selected oblique projections were obtained to clarify aneurysm anatomy at the discretion of the angiographer. All acquired DSA images were converted to internationally compatible DICOM files, and then the converted files were transferred to our server through a PACS.

\section{Image Analysis}

All imaging studies were evaluated for occlusion of the treated aneurysm, reconstruction of the parent artery (quality of visualization and measurable luminal diameter), and stent-related complications such as stent migration or in-stent stenosis. Studies from all patients for each imaging technique were placed into an anonymized folder on the PACS (ie, 3D-TOF MRA, CE-TR MRA, and DSA studies from all patient populations formed 3 anonymized folders). The entire original acquired data, including both source images (mask, subtracted, and unsubtracted images) and standard reformats (MIP and MPR) for each technique, were made available for review when evaluating that technique. Addi- 
Table 1: Quality of visualization of the reconstructed parent artery with the PED using 3D-TOF MRA and CE-TR MRA ${ }^{\mathrm{a}}$

\begin{tabular}{lccccc}
\hline Technique & Mean & SD & $\begin{array}{c}95 \% \mathrm{Cl} \\
\text { of Mean }\end{array}$ & $\begin{array}{c}\text { Comparison with } \\
\text { DSA }(\boldsymbol{P} \text { Value })\end{array}$ & $\begin{array}{c}\text { Comparison with } \\
\text { TR-MRA ( } \boldsymbol{P} \text { Value })\end{array}$ \\
\hline 3D-TOF MRA & 2.62 & 0.898 & $2.243-2.936$ & .0001 & .0001 \\
CE-TR MRA & 3.81 & 0.694 & $3.464-4.162$ & .344 & NA
\end{tabular}

Note:-NA indicates not applicable.

${ }^{a}$ Five-point grading scale from $0-4$ (0, very poor; 1, poor; 2, fair; 3, good; 4, excellent). DSA is considered the reference standard with a score of 4

Table 2: Measured luminal diameter of the reconstructed parent artery with the PED using 3D-TOF MRA and CE-TR MRA

\begin{tabular}{lccccc}
\hline \multicolumn{1}{c}{ Technique } & Mean $(\mathrm{mm})$ & SD & $\begin{array}{c}95 \% \mathrm{Cl} \\
\text { of Mean }\end{array}$ & $\begin{array}{c}\text { Comparison with } \\
\text { DSA }(\boldsymbol{P} \text { Value })\end{array}$ & $\begin{array}{c}\text { Comparison with } \\
\text { TR-MRA ( } \boldsymbol{P} \text { Value) }\end{array}$ \\
\hline 3D-TOF MRA & 2.108 & 0.964 & $1.718-2.497$ & .0001 & .0001 \\
CE-TR MRA & 3.137 & 0.546 & $2.910-3.351$ & .661 & NA \\
DSA & 4.096 & 0.687 & $3.819-4.374$ & NA & .661 \\
\hline
\end{tabular}

Note:-NA indicates not applicable.

Table 3: Overestimation of luminal stenosis of the reconstructed parent artery with the PED

\begin{tabular}{lccc}
\multicolumn{1}{c}{ Technique } & $\begin{array}{c}\text { Mean } \\
(\mathbf{m m})(\%)\end{array}$ & \multicolumn{1}{c}{ SD (\%) } & $\begin{array}{c}95 \% \mathrm{Cl} \\
\text { of Mean }\end{array}$ \\
\hline 3D-TOF MRA versus DSA & $1.992(71.8)$ & $0.998(52.7)$ & $0.036-3.948(31.6 \%-175 \%)$ \\
CE-TR MRA versus DSA & $0.965(26.6)$ & $0.497(12.7)$ & $0.01-1.941(1.7 \%-51.5 \%)$ \\
3D-TOF MRA versus CE-TR MRA & $1.027(50.1)$ & $0.776(58.8)$ & $0.495-2.55(65.1 \%-165 \%)$ \\
\hline
\end{tabular}

versus intensity of visualization was assessed by using $\chi^{2}$ analysis. Bland-Altman analysis was used to measure the numeric and percentage variation in the measurable luminal diameter. The significance of variation in the measurable luminal diameter between techniques was evaluated by using $\chi^{2}$ analysis. Correlation between the number of PEDs versus the measured lumen diameter was evaluated by using the Spearman correlation.

\section{RESULTS}

The sensitivity, specificity, and positive and negative predictive values of the $3 \mathrm{D}$ TOF MRA relative to DSA for confirmation of posttreatment aneurysm occlusion were $74 \%, 85 \%, 65 \%$, and $90 \%$, respectively. For CE-TR MRA relative to DSA, sensitivity, specificity, and positive and negative predictive values were $96 \%$, $85 \%, 92 \%$, and $92 \%$.

The mean quality of visualization in the arterial segment, reconstructed with a

tional reformats in challenging cases were created by the authors as needed by using the subtracted dataset. Images were reviewed by an experienced Certificate of Added Qualification-certified neuroradiologist (A.M.S.) in consensus with a neuroradiology fellow (S.R.B.). The various imaging techniques were reviewed separately to minimize the bias from knowledge of the results of the other imaging technique.

Images were evaluated for aneurysm occlusion following PED as a primary outcome of treatment. MR imaging demonstrating complete aneurysm occlusion in concordance with DSA was considered a true-positive. Angiographic quality of the reconstructed artery was assessed with respect to vessel visualization and susceptibility-mediated degradation compared with the adjacent native artery; the quality of visualization of the reconstructed artery by MRA was graded by using a 5-point scale from 0 to 4 as follows: 0 , extremely limited; 1 , poor; 2 , fair; 3 , good; 4 , excellent. The apparent luminal stenosis of the reconstructed artery was evaluated by measuring the maximal luminal diameter on both 3D-TOF MRA and CE-TR MRA. These findings were correlated with the quality of visualization and lumen diameter of the reconstructed artery on DSA as the reference standard. An experienced interventional neuroradiologist determined the clinical significance of discrepant findings between MRA techniques and DSA in the context of the particular patient study.

\section{Statistical Analysis}

Statistical analysis was performed by using SPSS software, Version 20 (IBM, Armonk, New York). The sensitivity, specificity, and positive and negative predictive values of 3D-TOF MRA and CE-TR MRA were measured. The quality of visualization of the reconstructed artery was compared between techniques with the Wilcoxon signed rank test. The correlation between the number of PEDs versus the quality of visualization was evaluated by using the Spearman correlation. Correlation between types of contrast
PED, on 3D-TOF MRA was $2.62 \pm 0.898$ and $3.81 \pm 0.694$ for CE-TR MRA (Table 1). The quality of visualization of the arterial segment reconstructed with a PED was superior on CE-TR MRA compared with 3D-TOF MRA $(P=.0001)$. Compared with the quality of visualization on DSA, both MRA techniques had inferior quality, with a significant difference for 3D-TOF MRA $(P=$ .0001) but not for CE-TR MRA $(P=.344)$.

The mean measurable luminal diameter of the arterial segment, reconstructed with the PED, on 3D-TOF MRA was $2.108 \pm$ 0.964 and $3.137 \pm 0.546$ on CE-TR MRA (Table 2). The measurable luminal diameter of the reconstructed artery on CE-TR MRA was higher compared with 3D-TOF MRA $(P=.0001)$. Compared with the luminal measurement on DSA, both MRA techniques overestimated the luminal stenosis within the PED, with a significant difference for 3D-TOF MRA $(P=.0001)$ but not for CE-TR MRA $(P=.661)$ (Table 3$)$.

Both 3D-TOF MRA and CE-TR MRA techniques showed a negative correlation between the number of PEDs used versus the intensity of visualization $\left(r_{=}-0.8\right.$ and -0.3 , respectively) and the number of PEDs used versus the measurable luminal diameter ( $r=-0.9$ and -0.6 , respectively) of the reconstructed artery. The type of contrast material caused no significant variation in the intensity of visualization of the reconstructed artery on CE-TR $\operatorname{MRA}(P=.684)$.

\section{DISCUSSION}

The PED provides flow diversion by an attenuated braided construction of a bimetallic design of $75 \%$ cobalt chromium and $25 \%$ platinum tungsten. The device has a 30\%-35\% metal surface area when fully deployed, which is substantially greater than the $6.5 \%-9 \%$ metal surface area coverage provided by the commercially available self-expanding intracranial microstents. ${ }^{2}$ The 

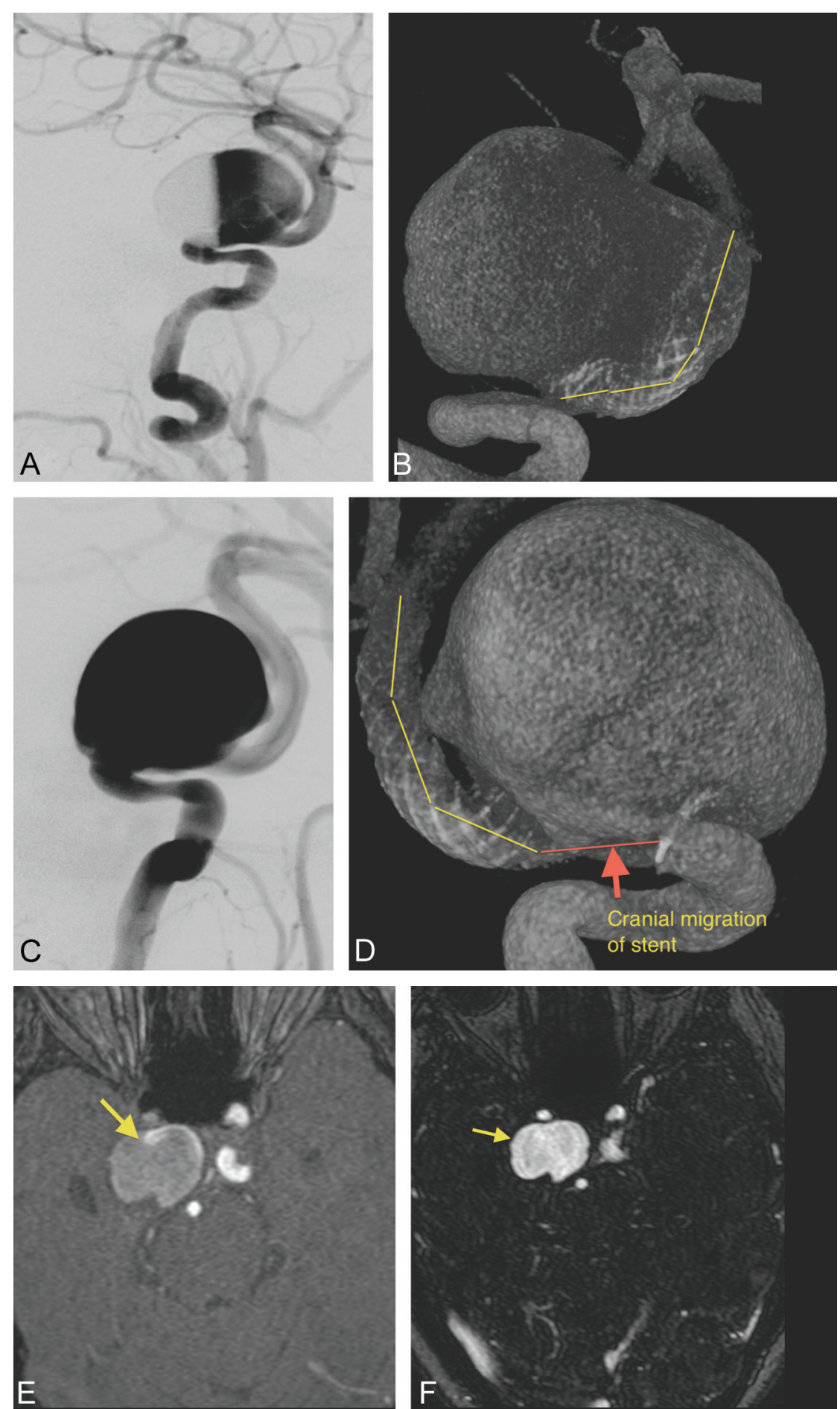

FIG 1. Stent migration with concordance between CE-TR MRA and DSA. A 56-year-old woman with a right cavernous carotid aneurysm. $A$, Immediate post-PED of the right cavernous ICA aneurysm with stasis. $B$, DSA shows the actual position of the PED after embolization (yellow line along stent course). C, Complete opacification of the aneurysm at 6-month DSA with no stasis or thrombus. D, DSA confirms distal migration (yellow line along stent course) resulting in reopening of the aneurysm neck (red line with red arrow). Superior demonstration of complete aneurysm opacification (yellow arrow) on CE-TR MRA (F) over 3D-TOF MRA (E) at 6-month follow-up. The hyperintensity in the aneurysm sac on TOF MRA (E) may represent either thrombus from intact PED or aneurysm refilling from PED migration (yellow arrow).

pooled sensitivity and specificity for evaluating complete aneurysm occlusion after coil embolization was $83 \%$ and $91 \%$ by using TOF-MRA and $87 \%$ and $92 \%$ with CE-MRA. ${ }^{12}$ CE-MRA was contributory in only $6 \%$ of the patients with treated intracranial aneurysms by using coil embolization. ${ }^{7}$ The results of MRA follow-up for coil-embolized aneurysms should not be directly applied to patients treated with the PED. In contradistinction to platinum coils, the larger bimetallic surface area coverage and radiofrequency shielding by the PED can result in a marked local signal void. ${ }^{13,14}$

Aneurysm residua, recurrence due to recanalization, in-stent stenosis due to intimal hyperplasia, stent occlusion from an organized thrombus, and stent migration must be evaluated by follow-up imaging in patients with parent vessel reconstruction. ${ }^{15,16}$ Shorter angiographic followup has been shown to be a negative predictor of aneurysm obliteration following PED. ${ }^{17}$ This finding highlights the importance of the immediate and long-term posttreatment follow-up in patients after PED.

Our results showed that the quality of visualization and measured luminal diameter of the reconstructed arterial segment on 3D-TOF MRA were significantly less than those on CE-TR MRA and DSA. These parameters were further adversely affected by the increased number of PEDs used for reconstruction. The inherent spatial resolution of 3D-TOF MRA in our imaging protocol is superior, with voxel reconstruction of $0.43 \times 0.43 \times 0.7 \mathrm{~mm}$ compared with CE-TR MRA with a larger interpolated voxel size of $0.48 \times 0.48 \times$ $0.7 \mathrm{~mm}$. We believe our findings on $3 \mathrm{D}$ TOF MRA, despite its better spatial resolution, are due to the signal loss from a combination of the inherent high metallic surface area of the PED and the predominant use in the proximal anterior intracranial circulation. Turbulent flow with intravoxel dephasing and/or slow flow with subsequent spin saturation can result in marked signal loss and can limit TOF MRA in tortuous vascular segments such as the cavernous or supraclinoid ICA. ${ }^{13,14,18,19}$ Among the patients constituting our study population, all except 2 subjects ( 1 with an anterior temporal artery aneurysm and 1 with a PICA aneurysm) underwent treatment of intracranial ICA aneurysms distributed between the intradural cavernous ICA and carotid terminus. Tortuosity and redundancy among these segments may expose such vulnerabilities in 3D-TOF MRA and likely account, in part, for the observed results. 

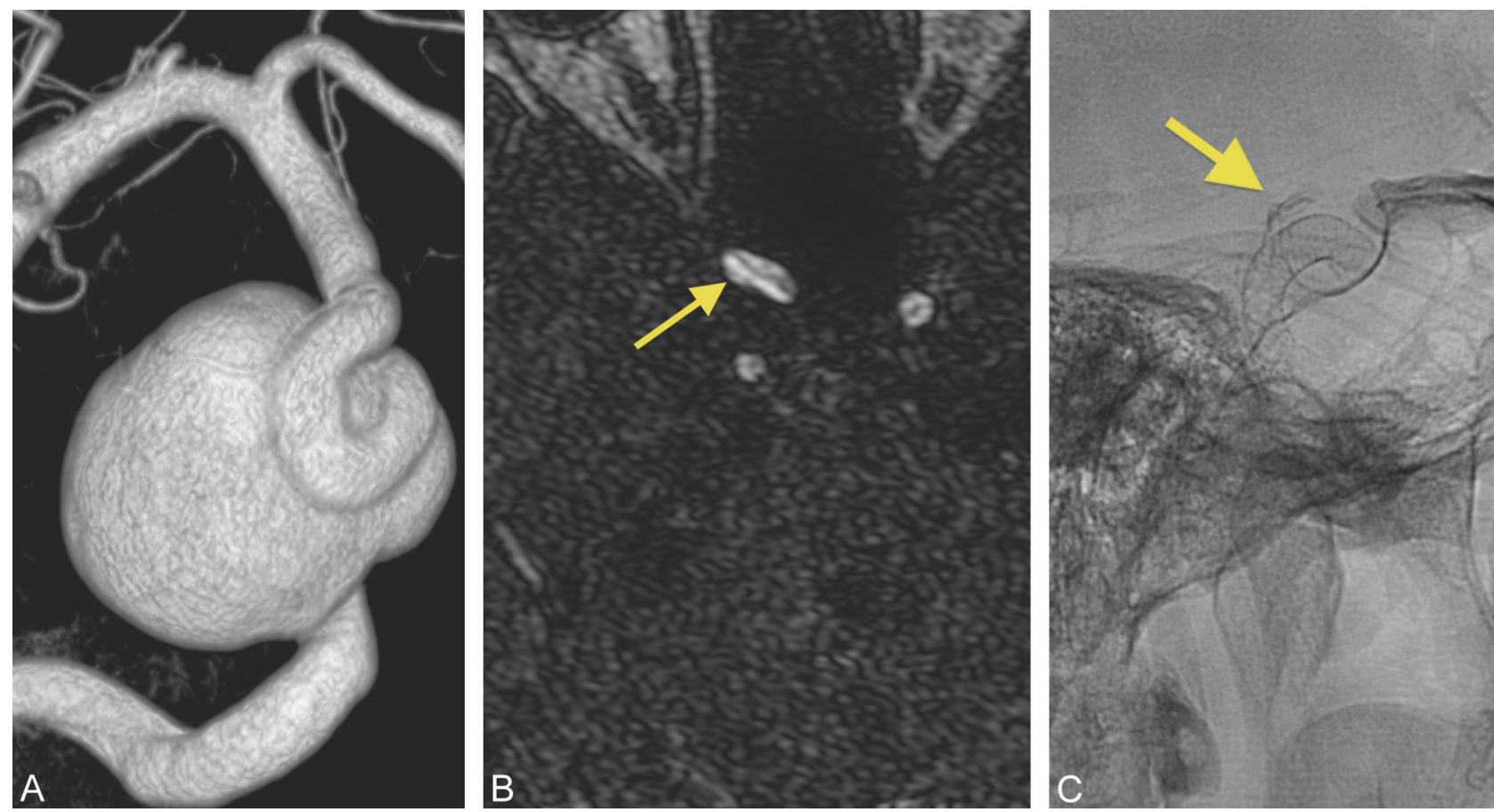

FIG 2. Discordance between CE-TR MRA and DSA. A 68-year-old woman with a right paraophthalmic aneurysm. A, Post-Pipeline embolization right paraophthalmic aneurysm. B, No residual neck is demonstrated (yellow arrow) on the 6-month follow-up CE-TR MRA. C, A thin crescentic residual neck measuring $1 \mathrm{~mm}$ deep (yellow arrow) is seen on the subsequent DSA.
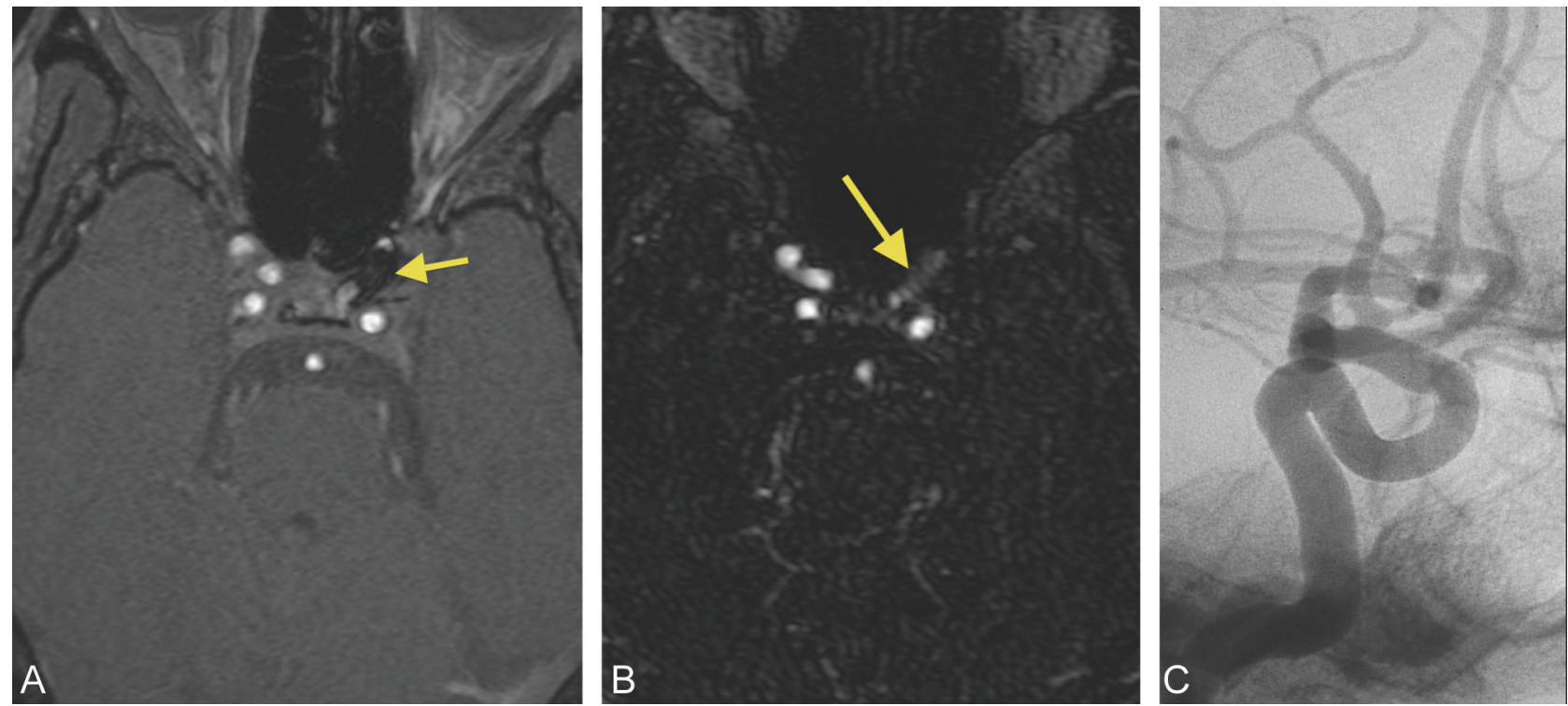

FIG 3. Overestimation of in-stent stenosis on MRA techniques. A 63-year-old woman with a left ophthalmic artery aneurysm. A, 3D-TOF MRA shows complete loss of flow-related enhancement in the PED (yellow arrow), suggestive of occlusion. B, Preserved enhancement within the PED with a narrowed lumen (yellow arrow) suggestive of in-stent stenosis. C, DSA demonstrates a normal-caliber ICA with no in-stent stenosis or occlusion.

3D-TOF MRA is further limited in its ability to differentiate thrombus from residual flow in treated aneurysms, both of which may exhibit intrinsic T1-weighted hyperintensity. Because the PED is designed for $85 \%$ reduction of blood circulation within the aneurysm, which induces thrombosis, ${ }^{20}$ this limitation decreases the diagnostic accuracy of 3D-TOF MRA compared with CE-TR MRA.

CE-TR MRA showed significantly superior quality of visualization and higher measurable luminal diameter of the recon- structed arterial segment compared with 3D-TOF MRA, a better reflection of DSA findings. CE-TR MRA uses precontrast mask images to subtract the background signal before contrast injection, so high signal intensity due to the T1 shortening effect of a thrombosed aneurysm is effectively eliminated. Signal loss related to turbulent flow or susceptibility artifacts may be partially overcome with conventional non-time-resolved CE-MRA; however, the limitations with this technique are venous contamination and enhancement of the aneurysm wall, potentially leading to false- 
positive results. ${ }^{11}$ While high-temporal-resolution and multiphasic MRA examinations are not critical for evaluation of the PED, they effectively prevent mistiming of the contrast bolus. Of note, none of the CE-TR MRA examinations were technical failures due to inadequate contrast-bolus timing. CE-TR MRA also would have potential benefit for cases with postprocedural rupture of cavernous segment aneurysms with arteriovenous fistula formation, though none were present in this study population. A single distal stent migration in 1 patient with refilling of the entire aneurysm was diagnosed on CE-TR MRA and was confirmed on DSA (Fig 1).

Small residual lumen and suboptimal projections on DSA were independently associated with discordance between intraarterial DSA and MR angiography. ${ }^{7}$ In our study group, 3 patients were nonconcordant between the CE-TR MRA and DSA. Two of 3 patients had false-positives, with erroneous diagnosis of complete aneurysm occlusion following PED. Subsequent DSA confirmed a thin crescentic filling in the aneurysm neck measuring 1 $\mathrm{mm}$ in depth (Fig 2). Findings in 1 of 3 patients were false-negative with minimal interstitial filling with an intra-aneurysmal coil pack on CE-TR MRA, which was demonstrated to be complete occlusion on the DSA performed on the same day. The superior spatial resolution of DSA compared with CE-TR MRA can explain the discordance between the techniques. DSA has a superior spatial resolution of $0.2 \times 0.2 \times 0.2 \mathrm{~mm}$ isotropic voxel size, ${ }^{21}$ compared with the $0.48 \times 0.48 \times 0.7 \mathrm{~mm}$ voxel size of CE-TR MRA, which increases the sensitivity for small 1-mm residual filling in the treated aneurysms. In each of these cases, the results did not affect immediate patient outcome in terms of repeat endovascular treatment or anticoagulation. Patients undergo the standard follow-up procedure of our institution: 6 months, 2 years, and 5 years' posttreatment with the PED.

Overestimation of in-stent stenosis was well-reported in the literature in both in vitro and in vivo studies by using 3D-TOF MRA techniques ${ }^{22,23}$ and on CE-TR MRA. ${ }^{11}$ Our study results are in agreement with the literature findings (Fig 3). 3D-TOF MRA overestimated the measurable lumen diameter of the PED by $72 \%$ in comparison with DSA or by $50 \%$ in comparison with CE-TR MRA. CE-TR MRA overestimated the measurable lumen diameter of the PED by only $27 \%$ in comparison with DSA. This overestimation is a definite limitation of both MRA techniques, because even small degrees of intimal hyperplasia and luminal narrowing, which are much more effectively evaluated by DSA (due to lack of artifactual apparent narrowing), can impact patient management. Indeed, the intrinsic spatial and temporal resolution of DSA is superior to MRA techniques. Incorporation of MRA techniques in the follow-up algorithm of PED to exclude a tiny residual/recurrent aneurysm filling or in-stent stenosis should be evaluated in light of clinical context, as this may affect the patient management on individual basis.

We acknowledge the limitations of this study. First, the retrospective nature of this study incorporates a learning curve in both the performance of the technique by technologists and the experience of the readers. The relatively small number of cases is also a limitation, and clinically significant discrepancies between the CE-TR MRA and DSA could emerge as a larger number of procedures are performed. In some cases, DSA was performed with knowledge of the MRA results, potentially influencing detection. Finally, while follow-up MRA and DSA were performed on the same day for most patients, in a few cases there was a time interval between the 2 examinations, which could result in disappearance or new emergence of contrast filling within an aneurysm that would introduce discrepancies between techniques not present if the studies had been performed on the same day.

\section{CONCLUSIONS}

CE-TR MRA can be used to noninvasively monitor intracranial aneurysms following flow diversion and parent vessel reconstruction with the PED. CE-TR MRA is superior to conventional 3DTOF MRA and has excellent concordance with DSA for detection of aneurysm occlusion and visualization of the reconstructed artery. Both MRA techniques evaluated overestimated apparent instent stenosis, which was worse on 3D-TOF MRA compared with CE-TR MRA.

\section{REFERENCES}

1. Molyneux AJ, Kerr RSC, Birks J, et al. Risk of recurrent subarachnoid haemorrhage, death, or dependence and standardised mortality ratios after clipping or coiling of an intracranial aneurysm in the International Subarachnoid Aneurysm Trial (ISAT): long-term follow-up. Lancet Neurol 2009;8:427-33

2. Fiorella D, Kelly ME, Turner R, et al. Endovascular treatment of cerebral aneurysms: current devices, emerging therapies and future technology for the management of cerebral aneurysms. Endovasc Today 2008;7:53-65

3. Kallmes DF, Ding YH, Dai D, et al. A second-generation, endoluminal, flow-disrupting device for treatment of saccular aneurysms. AJNR Am J Neuroradiol 2009;30:1153-58

4. Becske T, Kallmes DF, Saatci I, et al. Pipeline for uncoilable or failed aneurysms: results from a multicenter clinical trial. Radiology 2013;267:858-68

5. Delgado Almandoz JE, Crandall BM, Fease JL, et al. Successful endovascular treatment of three fusiform cerebral aneurysms with the Pipeline embolization device in a patient with dilating HIV vasculopathy. J Neurointerv Surg 2014;6:e12

6. Kaufmann TJ, Huston J 3rd, Mandrekar JN, et al. Complications of diagnostic cerebral angiography: evaluation of 19,826 consecutive patients. Radiology 2007;243:812-19

7. Schaafsma JD, Velthuis BK, Majoie CB, et al. Intracranial aneurysms treated with coil placement: test characteristics of follow-up MR angiography-multicenter study. Radiology 2010;256:209-18

8. Blackham KA, Passalacqua MA, Sandhu GS, et al. Applications of time-resolved MR angiography. AJR Am J Roentgenol 2011;196:W613-20

9. Cashen TA, Carr JC, Shin W, et al. Intracranial time-resolved contrast-enhanced MR angiography at 3T. AJNR Am J Neuroradiol 2006;27:822-29

10. Farb RI, Agid R, Willinsky RA, et al. Cranial dural arteriovenous fistula: diagnosis and classification with time-resolved MR angiography at 3T. AJNR Am J Neuroradiol 2009;30:1546-51

11. Choi JW, Roh HG, Moon W-J, et al. Time-resolved 3D contrastenhanced MRA on 3.0T: a non-invasive follow-up technique after stent-assisted coil embolization of the intracranial aneurysm. Korean J Radiol 2011;12:662-70

12. Kwee TC, Kwee RM. MR angiography in the follow-up of intracranial aneurysms treated with Guglielmi detachable coils: systematic review and meta-analysis. Neuroradiology 2007;49:703-13

13. Wall A, Kugel H, Bachman R, et al. 3.0 T vs. 1.5 T MR angiography: in vitro comparison of intravascular stent artifacts. J Magn Reson Imaging 2005;22:772-79

14. Wang Y, Truong TN, Yen C, et al. Quantitative evaluation of suscep- 
tibility and shielding effects of nitinol, platinum, cobalt-alloy, and stainless steel stents. Magn Reson Med 2003;49:972-76

15. Fiorella D, Albuquerque FC, Deshmukh VR, et al. Usefulness of the Neuroform stent for the treatment of cerebral aneurysms: results at initial (3-6-mo) follow-up. Neurosurgery 2005;56:1191-201, discussion 1201-02

16. Siddiqui MA, Bhattacharya J, Lindsay KW, et al. Horizontal stentassisted coil embolisation of wide-necked intracranial aneurysms with the Enterprise stent: a case series with early angiographic follow-up. Neuroradiology 2009;51:411-18

17. Jabbour P, Chalouhi N, Tjoumakaris S, et al. The Pipeline embolization device: learning curve and predictors of complications and aneurysm obliteration. Neurosurgery 2013;73:113-20, discussion 120

18. Majoie CB, Sprengers ME, van Rooij WJ, et al. MR angiography at 3T versus digital subtraction angiography in the follow-up of intracranial aneurysms treated with detachable coils. AJNR Am J Neuroradiol 2005;26:1349-56
19. Wallace RC, Karis JP, Partovi S, et al. Noninvasive imaging of treated cerebral aneurysms. Part I. MR angiographic follow-up of coiled aneurysms. AJNR Am J Neuroradiol 2007;28:1001-08

20. Byrne J, Szikora I. Flow diverters in the management of intracranial aneurysms: a review. EJMINT 2012:1225000057. http://www.ejmint. org/original-article/1225000057. Accessed March 19, 2014

21. Kaufmann TJ, Kallmes DF. Diagnostic cerebral angiography: archaic and complication-prone or here to stay for another 80 years? AJR Am J Roentgenol 2008;190:1435-37

22. Blum MB, Schmook M, Schernthaner R, et al. Quantification and detectability of in-stent stenosis with CT angiography and MR angiography in arterial stents in vitro. $A J R A m J$ Roentgenol 2007;189:1238-42

23. Lettau M, Sauer A, Heiland S, et al. Carotid artery stents: in vitro comparison of different stent designs and sizes using $\mathrm{CT}$ angiography and contrast-enhanced MR angiography at $1.5 \mathrm{~T}$ and 3T. AJNR Am J Neuroradiol 2009;30:1993-97 